\title{
On Accelerated Aging of Mechanical Assets in Distribution Systems with Renewable Generation
}

\author{
Aaqib Peerzada \\ Miroslav Begovic \\ Wesam Rohouma \\ Robert Balog \\ peerzada@tamu.edu_begovic@ece.tamu.edu wesam.rohouma@qatar.tamu.edu robert.balog@qatar.tamu.edu \\ Texas A\&M University, Department of ECE, College Station, TX, USA and Doha, Qatar
}

\begin{abstract}
The integration challenges associated with the widespread adoption of the photovoltaic generation can be divided into operational and the maintenance issues. Work done in recent years has addressed issues like voltage rise and unbalance. Less attention was directed to the maintenance challenges like accelerated aging of mechanically controlled voltage support assets under rapidly changing conditions. In particular, there is need for analysis on the mechanism of accelerated wear and tear of devices such as onload tap changers and capacitor banks exposed to rapid voltage fluctuations. This article focuses on developing aging device models and proposes a novel device, referred to as D-STATCOM, to reduce the impact of non-scheduled distributed generation on aging of mechanically-switched devices commonly used in distribution feeders.
\end{abstract}

\section{Introduction}

Over the past decade, the capacity of the photovoltaic (PV) generation in the electric grid has increased significantly. In 2017, the cumulative PV capacity was estimated to be $398 \mathrm{GW}$ approximately. More than half of the total PV capacity $(\approx 60 \%)$ is represented by the utility-scale projects, and the rest include residential, commercial, and the off-grid installations. At current rate of expansion, it is estimated that the global PV capacity would surpass $1,000 \mathrm{GW}$ in the year 2023 [1]. In the United States, the adoption of PV technology is supported by the policies of the federal government. The investment tax credit (ITC), also known as a federal solar tax credit, offers a $30 \%$ deduction on the cost of installing a PV system by way of federal taxes.

Grid integration of distributed non-scheduled energy resources (DER) such as PV generation presents several operational and maintenance issues. Some of the operational difficulties include the voltage rise on distribution feeders, reverse power flow on radial feeders, voltage and current unbalance, and malfunctioning (inadequacy) of conventional protection devices [2]. The maintenance challenges include increased mechanical stress on devices like On-Load Tap Changers (OLTCs), voltage regulators (VRs), and the capacitor banks. High penetration of DER significantly alters the feeder voltages, forcing the voltage control devices to operate more frequently. As presence of a PV system can affect the flow of active and reactive power on a distribution feeder, electric utilities might feel less motivated in ratifying the interconnect requests of customer-owned small generators. The distribution system operators make use of OLTCs, VRs, and capacitor banks to maintain an acceptable voltage profile across the feeder and to minimize active power losses. Many of those devices are mechanical in nature and rely on the local activation signals to perform. For example, the control setting of voltage regulators includes the time delay (TD), voltage set-point, and bandwidth. Similarly, for capacitor banks, discrete control of the reactive power output is implemented to improve the substation power factor.

Furthermore, the voltage regulators in most practical implementations make use of line drop compensation to regulate the secondary bus voltage. Operation of these devices involves the movement of a mechanical switch to output the desired voltage value and the power factor. The presence of PV generation may lead to an increase in the number of operations and subsequent wear and tear [3]. Multiple studies focused on evaluating the impact of solar PV intermittency on the operation of OLTCs and VRs suggest that an increase in the penetration of PV generation on a distribution feeder results in a nearlinear rise in the cumulative operations of the load tap changers [4],[5],[6]. However, the models used in these studies ignore the aging aspect of devices under conditions of increased electrical or mechanical stress. Furthermore, there exists a significant disparity in use of solar data across different studies. To adequately capture the impact of solar variability on the operation of load tap changers, it is essential to utilize highresolution data spread over a long time frame. To this end, the typical meteorological year (TMY-like) solar data with a time resolution of at least 1 minute, is 
recommended for any study which focusses on the solar PV integration challenges.

Since the variability of active (and reactive) power produced by DER negatively impacts reliability of the mechanical voltage control devices; it is important to develop the aging models of such devices and device components. A device aging model based on inverse power law and the Arrhenius model is given in [7],[8],[9]. The likelihood of failure is given by a twoparameter Weibull fit, which includes electrical, thermal, and mechanical stress. In [10], a transformer lifetime model based on a Bayesian method utilizing Perk's hazard function and Iowa curves is presented. Both of the models, however, lack the positive impact of device maintenance on the device lifetime.

In recent years, a lot of attention has been paid to address the operational challenges associated with PV integration. However, the impact on the mechanicallyswitched devices in terms of accelerated device aging and shortening of device lifetime has not been thoroughly investigated. Also, possible solutions in terms of solid-state power electronic devices, to alleviate such mechanical stress imposed by the intermittent PV generation on such devices have not been seriously considered, primarily due to their elevated cost. The objective of this article is to shed light on those issues and propose possible solutions.

\section{Problem Statement}

The tap position of load tap changers is a function of the load and the PV injections on the feeder. More specifically, at any instant $\tau$

$$
\alpha_{R j}(\tau)=f\left(y^{\tau}, P_{P V}^{\tau}\right)
$$

Where, $\alpha_{R j}(\tau)$ is the tap ratio of $j^{\text {th }}$ load tap changer at time instant $\tau, y^{\tau}=\left[P_{i}^{\tau} Q_{i}^{\tau}\right]^{T} ; i=1,2, \ldots, N$ is the vector of active and reactive load injections at time instant $\tau$ and $P_{P V}^{\tau}=\left[P_{P V k}^{\tau}\right] ; k=1,2, \ldots, L$ is the vector of PV injection at time instant $\tau$. Assuming the length of the planning period be $T$ and $\lambda$ the step size, the cumulative number of operations of $j^{\text {th }}$ load tap changer with $\Lambda$ as the step change in voltage is

$$
\zeta_{j}=\sum_{t=\tau}^{T} \frac{\alpha_{R j}(t)-\alpha_{R j}(t-\lambda)}{\Lambda}
$$

The increase in the cumulative operations of the tap changing devices under PV generation represents an increase in the mechanical stress on such devices. For the increased mechanical stress $M$,

$$
M=f\left(\Delta \zeta_{j}\right)=f\left(\zeta_{j}^{P V}-\zeta_{j}^{N o P V}\right)
$$

The overall objective of this work is to study the impact of (3) on the reduction in the reliability and shortening of the lifetime of mechanical voltage control assets under high penetration scenarios of PV generation. This provides a window to ascertain the cost of operating the voltage regulating equipment under conditions of accelerated aging due to the variability associated with PV generation.

\section{Tap Degradation Model}

The aging mechanism of a transformer depends on the reliability of essentially four transformer components. These include, 1) the reliability of paper winding insulation, 2) the reliability of transformer tank 3) the reliability of transformer bushings and 4) the reliability of load tap changers. The paper winding insulation is mostly impacted by the electrical and the thermal stress and is often modeled by making use of Arrhenius equation. The transformer tank is affected by corrosion, the impact of which can be reduced by employing proper maintenance procedures of the tank. The transformer bushings age due to the thermal stress and the operating load of the transformer. The degradation mechanism of the load tap changers includes asynchronous operation of switches, the formation of the carbon layer on the contacts and the higher operating frequency of the switches due to higher mechanical stresses imposed by the abnormal feeder voltages. The inverse power law dictates that for an electrical component exposed to a stress $M$, the life model is given by

$$
L_{P V}=L_{N o P V}\left(\frac{M_{P V}}{M_{N o P V}}\right)^{-n}
$$

where $n$ is the stress-coefficient, $S_{N o P V}$ is the scaleparameter corresponding to lower limit of stress i.e. operating stress in the absence of PV and $L_{N o P V}$ is the associated lifetime. Aging of a tap changer can be neglected at a stress level $M<M_{N o P V}$. Given a thermal stress $T$, the Arrhenius model takes the form

$$
L=L_{0}\left(\frac{M_{P V}}{M_{N o P V}}\right)^{-n} e^{-B T}, T=\frac{1}{\phi_{0}}-\frac{1}{\phi}
$$

In (5), $B$ is proportional to activation energy related to the main thermal degradation in the insulation, $T$ is the thermal stress, $\phi$ is the absolute temperature and $\phi_{0}$ is the reference temperature. The likelihood of failure at a given stress is given by a two parameter Weibull function

$P=1-\exp \left[-\left(\frac{M_{P V}}{M_{N o P V}}\right)^{\beta}\left(\frac{L_{P V}}{L_{N o P V}}\right)^{\frac{\beta}{n-b T}} e^{\frac{\beta B T}{n}-b T}\right]$

Considering only the mechanical stress imposed by the frequently changing feeder voltages and ignoring the thermal stress, the likelihood of failure can be expressed as 


$$
P=1-\exp \left[-\left(\frac{M_{P V}}{M_{N o P V}}\right)^{\beta}\left(\frac{L_{P V}}{L_{N o P V}}\right)^{\frac{\beta}{n}}\right]
$$

The coefficients $\beta$ and $n$ are obtained through experimental aging tests. In [7], $\beta$ and $n$ are determined by plotting the two lifetimes of a component against the two different stress conditions. If $L_{1}$ is the device lifetime in the absence of PV generation and $L_{2}$ is the device lifetime in the presence of stress and let $M_{1}$ and $M_{2}$ be the corresponding mechanical stress on the tap changers

$$
\begin{gathered}
n=\left(\frac{\log L_{1}-\log L_{2}}{\log S_{2}-\log S_{1}}\right) \\
\beta=\left(\frac{\log \left[\frac{\ln \left(1-P_{1}\right)}{\ln \left(1-P_{2}\right)}\right]}{\log L_{1}-\log L_{2}}\right)
\end{gathered}
$$

The following narrative addresses some practical experiences and attempts to determine the aging models of switching devices in electric power systems.

\section{Failure Statistics of OLTCs}

The transformer reliability working group founded in 1975, launched a survey in 1978 aimed at studying the lifetimes of transformers and reactors. The survey conducted is representative of the countries in CIGRE SC 12 [11]. The survey compiled data from more than 1000 failures that occurred between 1968-1978. Various distinctions were made to arrive at the failure rates of transformer populations. Some of them include the operating voltage, the transformer type, age and the presence of OLTC. Also, a distinction was made between forced outages and scheduled outages. Forced outages necessitate the transformer disconnection while for scheduled outages, the required maintenance could be planned at a later time. The survey concluded that the failure rate of a transformer generally increased with the voltage due to the reduced reliability of the winding paper insulation. When the failure rates were estimated in terms of device components, it was found that for distribution transformers, OLTCs contributed to the transformer failure more than any other component. In fact, out of the 702 failures in the substation transformers, 691 occurred in transformers equipped with OLTCs. The data in [11] suggests that more than $40 \%$ substation transformers failed owing to failure of OLTC, $19 \%$ failed due to the failure in windings and roughly about $12 \%$ failures resulted from the tank and dielectric fluid. In terms of the origin mechanisms of failure, mechanical failures account for roughly 55\% of the total failures, followed by dielectric and thermal failures. Mechanical failures also resulted in forced

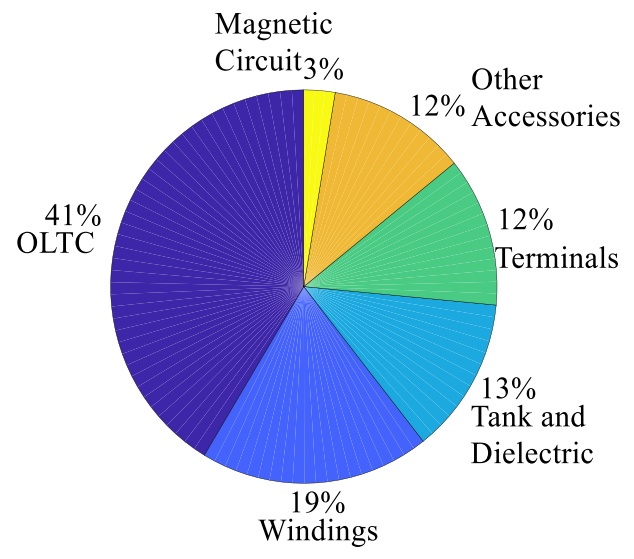

Figure 1 CIGRE SC 12 Failed Component Statistic

outages much more than thermal and dielectric based failures. The survey concluded that the failure in substation transformers occurred mostly due to the failure in the OLTCs.

In [12], the failure statistic of the 11 common wealth independent nations is presented. The data set includes failure modes of 5000 large power transformers with power rating of 100 MVA and above. For large power transformers, the primary cause of transformer failure was chiefly determined to be weak construction. Inadequate maintenance and low quality repair were also determined to be the leading causes contributing to the failure rate of power transformers.

In [13], the failure statistics of Escom network in South Africa is presented. The voltages from $88 \mathrm{kV}$ to $765 \mathrm{kV}$ and the transformers with power rating between 20 MVA and 800 MVA were considered. These voltage and power ranges includes distribution substation transformers as well as transmission power transformers. In the Escom network, the study in [13] identified six failure modes of transformers. These include lightning, core problems, tap-changer failures. General aging, short circuit problems and others. The study concludes that majority of the failures in distribution substation transformers were a result of tap changer initiated failures whereas in large power transformers, lightning and insulation problems at higher voltages contributed to the majority of the failures. The results of the survey in [13] are given in Figure 2.

Tap-changer-enabled transformers are still playing a major role in voltage regulation for radial distribution feeders. As such, it is important to study the reliability of a load tap-changer under adverse operating conditions. In recent years, many advances 


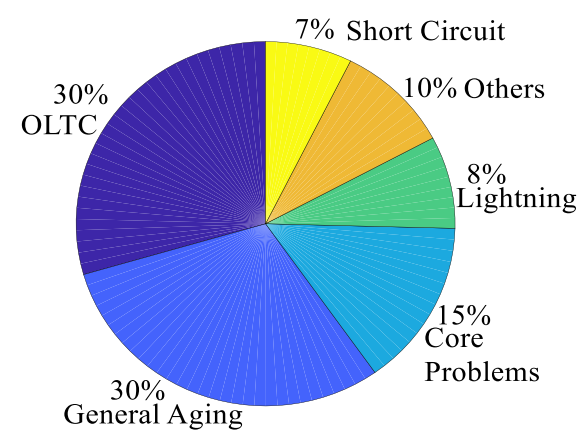

Figure 2 Eskom Network Failure Statistic of Transformers 20-100 MVA

have been made in the vacuum-type load tap-changers. However, the majority of the substation transformers are equipped with oil-type load tap-changers. Oil-type load tap-changers are negatively impacted by a higher frequency of operation due to the formation of carbon layer on the contacts of a tap-changer. As the frequency of operation increases it is more likely to encounter asynchronous operation between the diverter switch and the tap selector. The oil-type load tap changers generally require maintenance interventions between 50,000 and 100,000 operations. Given a transformer lifespan of 40 years, roughly five maintenance intervals are required for oil-type OLTCs. The vacuum type OLTCs on the other hand require less maintenance interventions. In [14], a statistical model for the tap-changer degradation is presented. The Weibull parameters $\alpha$ and $\beta$ for the tapchanger are 109 years and 2.4. The mean time to failure (MTTF) is reported as 97 years.

\section{Voltage Control Framework}

To properly address the question of quantification of degradation of mechanically-switched devices, it is important to ensure the optimal operation of such devices. The optimization of mechanical assets usually involves the determination of optimal device locations and settings. In the first step, the optimal locations for the devices are determined followed by the optimal values of device settings. The objective function could take the form of minimization of feeder losses or feeder voltage deviations. For the tapchanging devices, the optimal control of the tap position is sought, whereas in case of capacitor banks the decision variable of the optimization problem is the load switching level. The optimal dispatch of the mechanical voltage control devices is done on a slower-time scale (30-60 minutes), and the optimization problems are subjected to the constraints of 1) bus voltage magnitudes, 2) source power factor and 3) limits on the distribution line flows.

\subsection{On-Load Tap Changers}

The tap-changing devices in the IEEE 34 bus test feeder are the in-line step-type voltage regulators. Voltage regulators are modeled as single phase

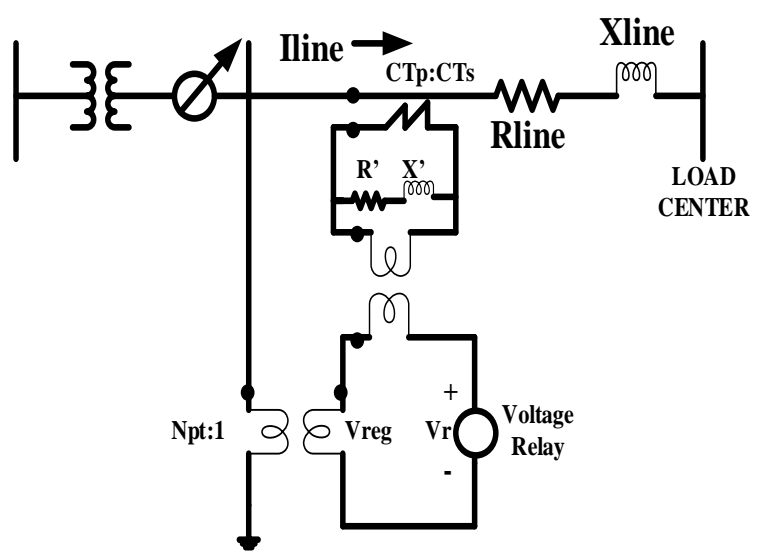

Figure 3 Line Drop Compensation Circuit

autotransformers with a nominal voltage regulation capability of $+/-10 \%$. This allows 32 taps with a minimum tap ratio of 0.9 and a maximum tap ratio of 1.1. The voltage regulators used in the study employ line drop compensation (see Figure 3 ) to estimate the load center voltage and the corresponding tap position. Only the forward power flow settings are provided and the voltage regulators are expected to provide regulation at the load center, which is usually the secondary of the autotransformer, in the range specified by the voltage set point and the bandwidth. The parameters $R^{\prime}$ and $X^{\prime}$ represent the equivalent impedance from the regulator to the load center. If the load center is the output of the regulator then the line drop compensator (LDC) settings are zero. The LDC settings include the ratios of the potential and the current transformer and the parameters $R^{\prime}$ and $X^{\prime}$. These parameters depend on the line impedance between the voltage regulator and load center, the CT and PT ratio and can be expressed as

$$
R^{\prime}+j X^{\prime}=\frac{Z_{\text {line }} C T p}{N p t}
$$

where, $Z_{\text {line }}=R_{\text {line }}+j X_{\text {line }}$ is the line impedance, CTp is the rated current from the substation and $N p t$ is the ratio of the potential transformer. The transformer tap position is determined from the unregulated load center voltage and the tap-changer bandwidth setting

$$
\operatorname{Tap}_{j}(\tau)=\operatorname{Int}\left[\frac{\left|\left(V_{\text {set }}-W / 2\right)\right|-\left|V^{u r}(\tau)\right|}{\Lambda}\right]
$$


where, $\operatorname{Tap}_{j}(\tau)$ is the tap position of the $j^{\text {th }}$ voltage regulator at instant $\tau, V_{\text {set }}$ is the voltage set-point of the $j^{\text {th }}$ voltage regulator, $W$ is the bandwidth and $V^{u r}(\tau)$ is the unregulated load center voltage at time instant, $\tau$ and

The voltage regulator positions are determined by observing the bus voltages and identifying the locations where the voltage first drops below the acceptable minimum value of 0.95 p.u. Successive snapshot power flow simulations are used to determine the positions of other voltage regulators. The simulation-based algorithm to determine the optimal voltage regulator positions is

- Run a snapshot power simulation at peak load and identify the location closest to the substation with voltage, $V_{i}<V_{\min }$.

- Place a voltage regulator at the location with tap position set to optimal value based on the unregulated bus voltages.

- With a voltage regulator at this location and tap at optimal position, run successive snapshot power flow simulations and identify the next closest bus to the substation with voltage less than $V_{\min }$.

- When all the voltages are in the range 0.951.05 , stop. Otherwise repeat the steps.

The optimal tap control of voltage regulators is accomplished by considering the objective function of the form

$$
\text { maximize } \sum_{(a, b) \in T} p_{a b}^{u r}(\tau)-\sum_{(a, b) \in T} p_{a b}^{r}(\tau)
$$

where, $T$ is the set of transmission links, $p_{a b}^{u r}(\tau)$ is the active power loss in the transmission link $(a, b)$ of the unregulated feeder at time instant $\tau$ and $p_{a b}^{r}(\tau)$ is the active power loss in the transmission link $(a, b)$ of the regulated feeder at time instant $\tau$. The active power loss can be expressed as

$$
p_{a b}(\tau)=\left|I_{a b}\right|^{2} r_{a b}=\frac{P_{a b}^{2}(\tau)+Q_{a b}^{2}(\tau)}{\left|V_{b}(\tau)\right|^{2}} r_{a b}
$$

The objective function in (12) can be equivalently expressed as

$$
\begin{aligned}
& \text { minimize } \sum_{(a, b) \in T} p_{a b}^{r}(\tau)=\sum_{(a, b) \in T}\left|I_{a b}^{r}\right|^{2} r_{a b} \\
& \text { subject to } \quad V_{\min } \leq V_{i}^{r}(\tau) \leq V_{\max } \\
& p f_{s s} \geq p f_{\text {min }} \\
& \left|F_{a b}\right| \leq\left|F_{a b}\right|_{\text {max }}
\end{aligned}
$$

Where, $p f_{s s}$ is the substation power factor and $F_{a b}$ is the flow on the transmission link $(a, b)$. At this point a simplifying assumption of constant power loads is made to derive the minimizing solution of (14). The minimizing solution is

$$
\operatorname{maximize}\left|V_{b}^{r}(\tau)\right|
$$

The maximum bus voltage cannot exceed the upper ANSI limit of 1.05 p.u. Let us represent it as $V_{\max }$.

$$
\left|V_{b}^{r}(\tau)\right|=V_{\max }
$$

With $V_{\max }$ as the maximum bus voltage, the optimal tap ratio of $j^{\text {th }}$ voltage regulator at time instant $\tau$ is [15]

$$
\alpha_{R j}^{*}(\tau)=\frac{V_{\max }}{\left|V^{u r}(\tau)\right|}
$$

where, $V^{u r}(\tau)$ is the unregulated load center voltage. The optimal tap position of a tap-changer can be expressed in terms of optimal tap ratio as

$$
\operatorname{Tap}_{j}^{*}(\tau)=\left\{\begin{array}{l}
\frac{1-\alpha_{R j}^{*}(\tau)}{\Lambda} ; \alpha_{R j}^{*} \in\left[\alpha_{R j, \min }, 1\right) \\
\frac{\alpha_{R j}^{*}(\tau)-1}{\Lambda} ; \alpha_{R j}^{*} \in\left(1, \alpha_{R j, \max }\right]
\end{array}\right.
$$

If $\alpha_{R j}^{*}(\tau)=1$, the optimal tap position is 0 , which is at the middle of tap range. When $\alpha_{R j}^{*} \in\left[\alpha_{R j, \min }, 1\right)$, the tap position is raised to increase the load center voltage and when $\alpha_{R j}^{*} \in\left(1, \alpha_{R j, \max }\right]$, the tap position is lowered to decrease the load center voltage.

\subsection{Capacitor Banks}

The capacitor banks are installed on the feeder for the purpose of improving the substation power factor. The capacitor banks serve to provide the reactive power demand of the loads thereby reducing the reactive power flow from the substation. Since the active and reactive load injections on the feeder are a function of time, and the capacitor banks can be controlled in a discrete fashion, it is essential to determine the load switch-on level of the capacitor banks. The optimization of capacitor banks involves the determination of optimal locations and the optimal control setting of the capacitor. To determine the optimal locations of the capacitor banks, the internal capacitor placement routine of OpenDSS is utilized which is based on the minimization of the objective function in (14). The typical capacity of the capacitor bank size is $300 \mathrm{kVAr}$, which is a standard bank at the nominal voltage of $24.9 \mathrm{kV}$. The discrete control of the capacitor is given by

$$
q_{c x}(\tau)=\left\{\begin{array}{c}
0 ; y \leq y^{S} \\
q_{c x}^{0}\left|V_{i}^{r}(y)\right|^{2} ; y>y^{S}
\end{array}\right.
$$

where, $q_{c x}(\tau)$ is the reactive power generated by the shunt capacitor at time instant $\tau, q_{c x}^{0}$ is the reactive power generated by the shunt capacitor at bus $i$, when 
$\left|V_{i}^{r}\right|=1, y$ is the vector of active and reactive load injections and $y^{S}$ is the vector of active and reactive load injections at which the capacitor bank is switched on/off. The optimal load switching vector $y^{S}$ is given by the intersection of the unregulated and regulated active power loss curves. Alternatively, at $y^{S}$

$$
\sum_{(a, b) \in T} p_{a b}^{u r}(y)=\sum_{(a, b) \in T} p_{a b}^{r}(y)
$$

The capacitor placement and control algorithm can be summarized as

- At the peak load and with an assumed bank size, determine the optimal locations of the capacitor banks.

- The optimal number of banks required is given by the figure of merit of the optimization problem. If $h$ is the figure of merit and $n_{c}$ is the optimal number of capacitor banks, then as $n \rightarrow n_{c} \Rightarrow h \rightarrow 0$.

- Decrement the load to determine $y^{S}$; the optimal bank switch-off/on level.

Figure 4 shows the IEEE 34 bus test feeder with the optimal locations and number of the mechanical voltage control assets.

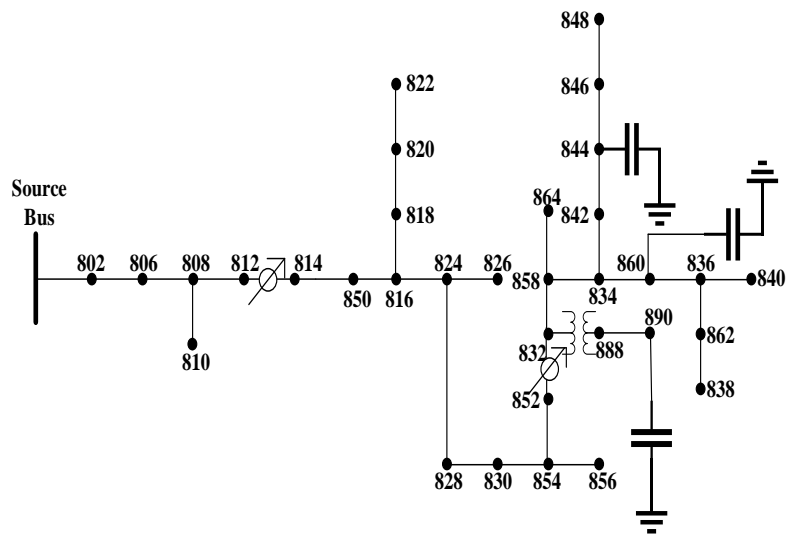

Figure 4 Optimized Mechanical Assets of IEEE 34 Test Feeder

Figure 5 and Figure 6 show the kW loss curves with and without PV generation. In Figure 5, the active power loss increases with the increase in feeder load and reaches a value of nearly $350 \mathrm{~kW}$ at peak feeder load, represented by the load multiplier, $\lambda=1$. When the feeder is compensated with the addition of three phase capacitor banks, the active power loss is more than in the uncompensated case, in the interval $0<$ $\lambda<0.58$. For $\lambda>0.58$, the active power loss is less than the uncompensated case. From (20), if $\lambda^{S}$ is the optimal load switching multiplier, then the optimal load switching level for the capacitor banks is

$$
y^{S}=\lambda^{S}\left[\mathbf{1}^{T} y^{P}\right] ; y^{P}=\left[P_{i}^{P} Q_{i}^{P}\right]^{T} ; i=1,2, \ldots, N
$$

where, $y^{P}$ is the column vector of peak load injections at all the load buses. With PV on the distribution feeder, the active power loss curves are convex functions of the feeder load as before, but the feeder loss decreases as the feeder load increases till $\lambda=\lambda^{S}$ in the uncompensated case.

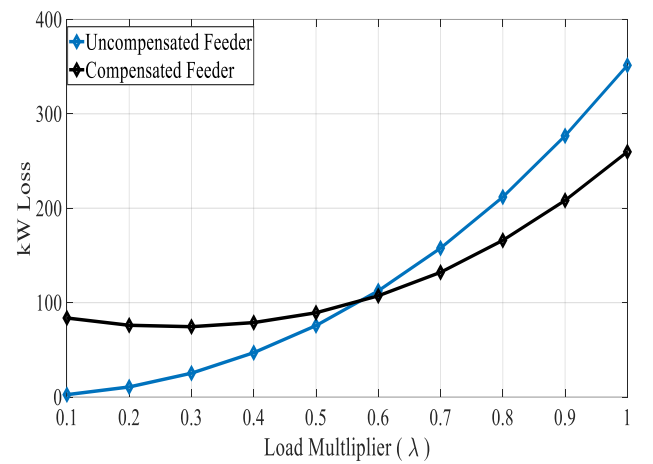

Figure 5 Active Power Loss Curves (No PV)

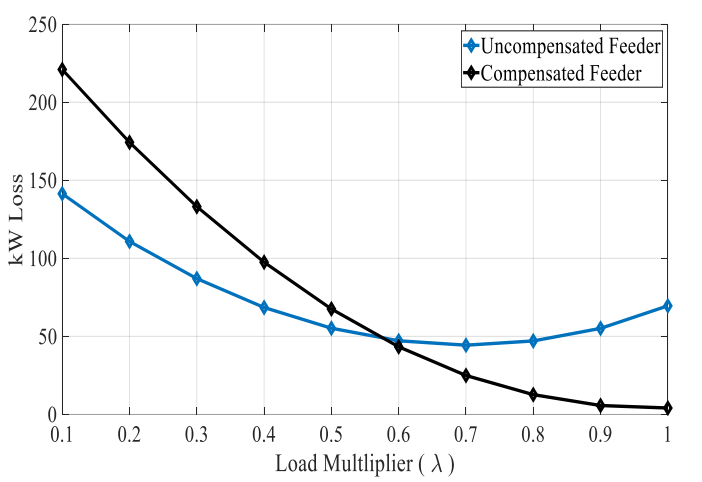

Figure 6 Active Power Loss Curves ( PV)

A similar behavior can be observed in the compensated case, except that the feeder losses continue to decrease even when $\lambda>\lambda^{S}$. The decrease in the feeder active losses with the increase in feeder load is explained by the fact that at lightly loaded conditions, the PV generation exceeds the overall feeder demand which increases the flow in the transmission links, thereby incurring high $I^{2} r$ losses. As the load increases, the flow in the transmission links decreases as the excess flow is taken by the increased power demand on the feeder.

\section{Modeling of Loads and Load Profiles}

For the purpose of carrying out year-long time series simulations, the feeder loads are divided into four categories based on their nominal values. The 
classification is given in Table 1. A combination of different load models is used for the quasi-static time series simulations (QSTS). This is done to better represent the diversity found in the loads on $\mathrm{MV} / \mathrm{LV}$ long radial distribution feeders.

\section{Table 1Classification of Feeder Loads}

\begin{tabular}{|c|c|}
\hline Load Class & Nominal kW Range \\
\hline $\begin{array}{c}\text { Small Dwelling (Studio } \\
\text { Apartment) }\end{array}$ & $P \leq 3 \mathrm{~kW}$ \\
\hline $\begin{array}{c}\text { Large Dwelling (House } \\
\text { with multiple } \\
\text { bedrooms) }\end{array}$ & $3 \mathrm{~kW}<P \leq 10 \mathrm{~kW}$ \\
\hline $\begin{array}{c}\text { Small Scale } \\
\text { Commercial }\end{array}$ & $10 \mathrm{~kW}<P \leq 25 \mathrm{~kW}$ \\
\hline $\begin{array}{c}\text { Medium Scale } \\
\text { Commercial }\end{array}$ & $25 \mathrm{~kW}<P \leq 100 \mathrm{~kW}$ \\
\hline $\begin{array}{c}\text { Large Scale } \\
\text { Commercial }\end{array}$ & $100 \mathrm{~kW}<P$ \\
\end{tabular}

Different loads models used include constant power loads, constant impedance loads, constant current loads and voltage-dependent loads. The use of such models is consistent with the test feeder specifications developed by the working group of the IEEE distribution sub-committee [16]. The voltagedependent loads are modeled as

$$
\begin{aligned}
& \frac{P(V)}{P_{0}}=\left(\frac{V}{V_{0}}\right)^{p} \\
& \frac{Q(V)}{Q_{0}}=\left(\frac{V}{V_{0}}\right)^{q}
\end{aligned}
$$

The parameter $p$ defines the relationship between the voltage $(V)$ and the active power $(P) . P_{0}$ is the nominal power of the load at the base voltage $V_{0}$. Similarly, $q$ defines the relationship between the voltage $(V)$ and the reactive $\operatorname{power}(Q) . Q_{0}$ is the nominal reactive power of the load at the base voltage $V_{0}$. The typical values of $p$ and $q$ range from $0.4-0.8$ and 2-3 respectively.

The year-long variation of loads is modeled according to the annual load change patterns developed by the United States Department of Energy (DOE). The OpenEI database [17] is a collection of residential and commercial loads across different locations in the United States. The data sets have a time resolution of 1 hour and are based on the Building America House Simulation Protocols which use Residential Energy Consumption Survey (RECS) for statistical references of building types by location. The commercial load data is based on the DOE commercial reference building models. The sub-hourly values are captured by means of linear interpolation. Since the
IEEE 34 bus test feeder is located in the state of Arizona, the annual load profiles of reference residential and commercial buildings representing Arizona are extracted from the OpenEI database. The loads on the IEEE 34 test feeder are decoupled in the sense that each individual load is assigned a unique annual variation, in compliance with its load class, as defined in Table 1. The load profiles are then normalized by the nominal demand to obtain an array of load multipliers that dictate the load behavior over the course of the planning period. This process results in a load behavior which is uncorrelated and hence representative of the real-world conditions.

\section{Impact of Data Sampling Rate}

The IEEE 34 bus test feeder is utilized to study the impact of solar variability on the mechanical voltage control assets. A high penetration, proportionaldistributed PV configuration is chosen to induce maximum operational stresses on the mechanical switches of tap-changers. The PV penetration is defined is given by

$\% P_{P V}=\frac{\sum_{k=1}^{L} P_{P V k}^{P}}{\psi} ; \psi \in y^{P} \cap\left[P_{i}^{P}\right] ; i=1, \ldots, N(24$

where $P_{P V k}^{P}$ is the peak generation of $k^{\text {th }} \mathrm{PV}$ system, $y^{P}$ is the vector of peak active and reactive load injections and $P_{i}^{P}$ are the peak active power load injections. $N$ and $L$ represent the number of buses and the number of PV systems on the feeder respectively. The output of a PV system is a function of solar irradiance incident on the solar panel, the ambient temperature and the panel orientation. To model a real world distribution of residential and commercial PV installations, a range of PV system orientation is used. The PV system orientation comprises of the PV panel tilt angle with respect to the horizontal surface and the direction in which the solar panel is facing, also referred to as the panel azimuth. It is reasonable to assume some variation in the tilt and the azimuth angles in a given set of PV installations on a distribution feeder.

For the purpose of demonstrating the impact of rooftop PV system orientation data in residential feeders, we assume a uniform distribution for the PV tilt angle and the PV panel azimuth angle. The common roof pitch angle in the United States is in the range of 4/12-9/12 which corresponds to pitch angle of 18.43-36.87 degrees. For the PV panel azimuth, a truncated uniform distribution in the range of 135-225 degrees from the true north is used. This choice of PV panel azimuth is justified because the IEEE 34 test feeder is located in the northern hemisphere and for a PV system in the northern hemisphere, the optimal 
azimuth angle is 180 degrees south. Given a tilt and the azimuth for a PV system, the tilted irradiance estimated by the transposition model [18]

$$
E(\beta)=E_{D N I} \cos \theta+E_{D H I} R_{d}+\rho E_{G H I} R_{r}
$$

where, $E_{D N I}, E_{D H I}, E_{G H I}$ refer to the direct normal irradiance, diffused horizontal irradiance and global horizontal irradiance, all in $W / \mathrm{m}^{2}$. The parameters $\beta$ and $\theta$ are the tilt angle and the angle of incidence respectively. The factors $R_{d}$ and $R_{r}$ are functions of the tilt angle, $\beta$.

The data for the $E_{D N I}, E_{D H I}$ and $E_{G H I}$ with a time resolution of 1 minute, 5 minutes and 1 hour are obtained from the NREL database. The high resolution (1 minute and 5 minute) data are the non-TMY data while the low resolution (1 hour) is the TMY3 data. The TMY data set represents typical weather conditions at a location over a longer period of time. The TMY3 data set is representative of the weather and solar data from 1976-2005. While the use of TMY3 data is recommended, since it represents typical weather conditions averaged over a longer period of time, it is not very suitable for studies involving solar conversion systems due to the low resolution. One possibility is to synthesize a data set with a high temporal resolution based on the low resolution TMY3 solar data. Such synthesis is possible through Markov chain modeling techniques. In this work, however, we attempt to study the difference in the distribution feeder response to the incoming solar irradiance with different temporal resolutions. We make no attempt to synthesize a high resolution data set from a low resolution data set. Figures 7,8 and 9 show the variation in tap position of the voltage regulator at 852-832 (see Figure 4 ) in response to solar irradiance with different temporal resolutions for a period of one day.

The solar irradiance with a time resolution of 1 minute is able to capture cloud transients in a higher detail than a 5 minute sampled irradiance pattern.

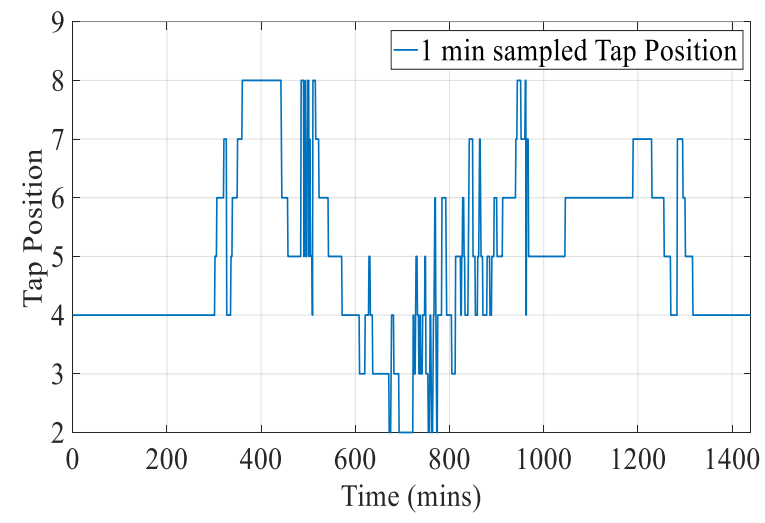

Figure 7 Tap Position of Reg 2A with 1 minute sampled Irradiance
This is reflected in the rapid movement of the regulator tap position in response of 1 minute sampled irradiance as compared with the 5 minute or the TMY3 irradiance. The standard time delay in OLTCs as quoted by various manufacturers is 30 seconds. For regulators which are placed downstream from the regulator closest to the substation transformer, the industry practice is to add a 15 second delay on top of the 30 second delay to initiate the tap action. In this work, the time delay for the voltage regulator at 812 814 is 30 seconds and the time delay for the voltage regulator at $852-832$ is set at 45 seconds. Based on the time delay of the OLTCs it is preferable to use the solar irradiance with a temporal resolution of at least 1 minute to accurately capture the operational stresses imposed on the OLTCs due to cloud transients.

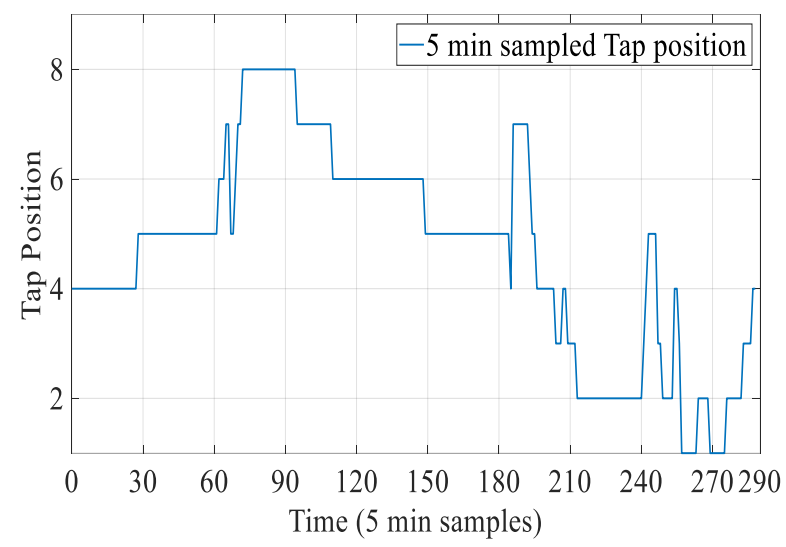

Figure 8 Tap Position of Reg 2A with 5 minute sampled Irradiance

The annual of tap operations for different temporal resolutions of the solar irradiance incident on the panels is given in Table 2 . From the numbers in Table 2 there is a significant difference between the number of tap operations registered in case of 1 minute sampled solar irradiance as opposed to the 5 minute sampled or a 1 hour sampled TMY3 data. However, the difference between the 5 minute sampled data and the TMY3 data is not so significant.

Table 2 Cumulative Tap Operations

\begin{tabular}{|c|c|c|c|}
\hline $\begin{array}{c}\text { Voltage } \\
\text { Regulator }\end{array}$ & $1 \mathrm{~min}$ & $5 \mathrm{~min}$ & $\begin{array}{c}1 \text { hour } \\
\text { (TMY3) }\end{array}$ \\
\hline $1-\mathrm{A}$ & 24194 & 13855 & 11552 \\
\hline $1-\mathrm{B}$ & 11816 & 7529 & 6666 \\
\hline $1-\mathrm{C}$ & 13698 & 8539 & 7470 \\
\hline $2-\mathrm{A}$ & 24089 & 11264 & 8586 \\
\hline $2-\mathrm{B}$ & 20850 & 10147 & 8100 \\
\hline $2-\mathrm{C}$ & 21298 & 10507 & 8136 \\
\hline
\end{tabular}




\section{D-STATCOM: Alternative Approach}

The investment in reactive support, one that offers continuous control adjustment and a longer service life, is the most promising solution to combat the accelerated wear and tear of the mechanical voltage control assets in the presence of PV generation

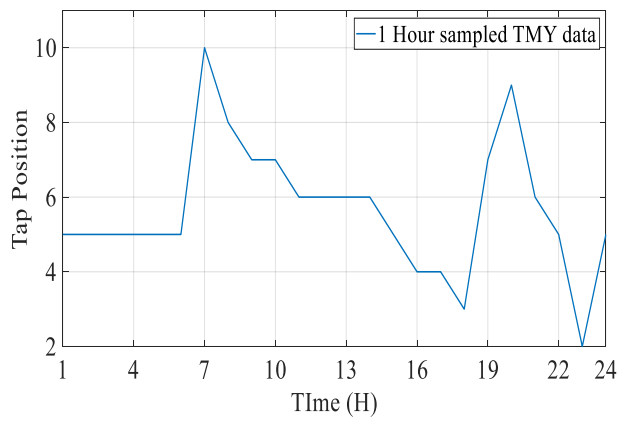

Figure 9 Tap Position of Reg 2A with 1 hour
sampled Irradiance

Such a solid-state based power electronic device offers fast and continuous control of the reactive power output. The D-STATCOM can be interfaced with the $\mathrm{MV} / \mathrm{LV}$ distribution feeder and can be operated in either power factor correction (PFC) mode or voltage regulation (VR) mode. In the PFC mode, the compensator cancels the reactive demand of the load at the bus where the compensator is connected by supplying the required reactive power, thus eliminating the need of supplying the reactive power from the source or upstream network. A range of power factors can be achieved at any load bus by adjusting the reactive power output of the compensator. On the other hand, if the system objective is to prevent the excessive tap operations of the tap-changers, the D-STATCOM can be run in VR mode. In this mode, the D-STATCOM is expected to autonomously determine the amount of VARs to be produced or consumed, to maintain the bus voltage within a specified bandwidth.

Voltage regulation by D-STATCOM is accomplished by making use of a proportional-integral (PI) controller to minimize the mismatch between the reference voltage and the measured bus voltage. The voltage regulation model is based on the reactive power mismatch equations. Let $V_{p c c}$ be the voltage at the point of common coupling and $V_{\text {conv }}$ be the converter bus voltage. The reactive power mismatch equations are

$\left[\begin{array}{c}Q-V_{p c c} I \sin \left(\theta_{V_{p c c}}-\theta_{I}\right) \\ Q+\left|V_{p c c}\right|^{2} B-\left|V^{\prime}\right| G \sin \delta+\left|V^{\prime}\right| B \cos \delta\end{array}\right]=0(26)$ where, $\left|V^{\prime}\right|=\left|V_{p c c}\right|\left|V_{c o n v}\right|$ and $\delta=\theta_{V_{p c c}}-\theta_{V_{c o n v}}$. In (26), $Q$ is the reactive power exchanged between the converter and the load bus, $G$ and $B$ are the line conductance and susceptance respectively. The operation of the D-STATCOM is coordinated with the voltage regulators by setting the reference voltage of the D-STATCOM equal to the voltage set-point of the voltage regulators. The voltage regulators will adjust the tap position whenever the load center voltage falls out of the specified bandwidth, after a certain time delay. The D-STATCOM is interfaced with the load bus 890 . The lateral $888-890$ experiences a significant drop in voltage mainly due to the heavy load at bus 890 and the long length of the line connecting buses 888 and 890 . This results in abnormally low voltages at bus 890 , and hence in need of voltage support to bring the phase voltages within compliance.

With a D-STATCOM at the far end of feeder and reference voltage set equal to the voltage set-point of the nearest upstream voltage regulator (1.03 p.u), QSTS simulations are initiated to study the interaction of the D-STATCOM and the load tap-changer. Figure 10 plots the bus 890 voltage with and without DSTATCOM enabled. It is clear that the presence of a continuously adjustable reactive support allows for a much better control of bus voltage than discrete mechanical action of the tap-changing devices. Since the action of tap-changers depends on the voltage variation, the D-STATCOM is a very attractive solution at limiting the excessive tap operations of such devices or even eliminate the need of mechanically-switched devices.

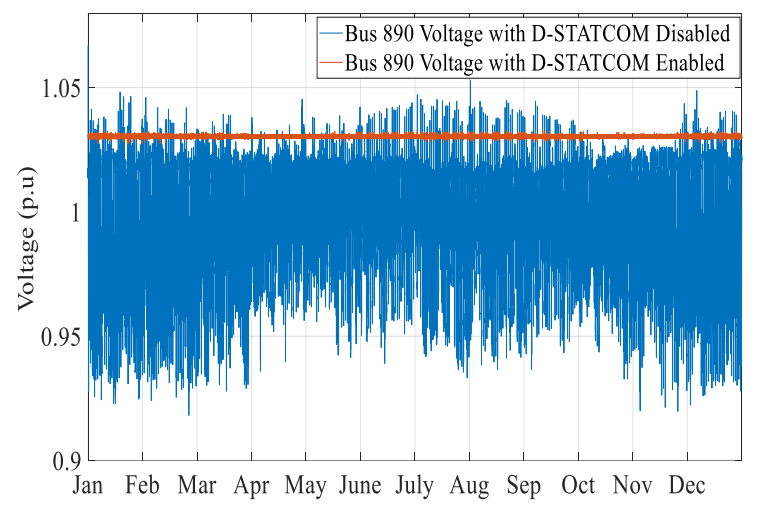

Figure 10 Voltage Profile of Bus 890

An alternate solution to reduce the degradation of the tap-changers is the volt-var control (VVC) of the smart inverters which can be programmed to deliver reactive power in addition to active power. However, the voltage regulation capability of a smart PV inverter is limited by the available volt-ampere reactive capacity. More specifically, at any instant $\tau$, the available reactive power is 


$$
Q_{\text {available }}(\tau)=\sqrt{S_{\text {rated }}^{2}-P_{\text {inv }}^{2}(\tau)}
$$

The operation of a D-STATCOM on the other hand is not limited by the active power capability of the device. This enables the D-STATCOM to maintain a quasi-constant bus voltage under highly variable PV generation as opposed to a smart PV inverter. Table 3 lists the annual cumulative tap operations of all the tap-changers with and without a D-STATCOM.. The last column of Table 3 contains the cumulative annual tap operations of tap-changers when a coordinated fleet of PV inverters is used for regulating PCC voltages. It can be observed that a D-STATCOM is able to achieve a larger reduction in the degradation of the tap-changing devices as opposed to a fleet of PV inverters.

\section{Table 3 Impact of D-STATCOM and PV inverter on Tap Activity}

\begin{tabular}{|c|c|c|c|c|}
\hline VR & $\begin{array}{c}\text { D- } \\
\text { STACOM } \\
\text { Disabled }\end{array}$ & $\begin{array}{c}\text { D- } \\
\text { STATCOM } \\
\text { Enabled }\end{array}$ & $\begin{array}{c}\text { \% } \\
\text { decrease }\end{array}$ & $\begin{array}{l}\text { PV } \\
\text { Inverter } \\
\text { Enabled }\end{array}$ \\
\hline 1-A & 24144 & 16364 & $\mathbf{3 2 . 2}$ & 16400 \\
\hline 1-B & 11816 & 5823 & $\mathbf{5 0 . 7}$ & 8800 \\
\hline 1-C & 13968 & 7149 & $\mathbf{4 8 . 8}$ & 10200 \\
\hline 2-A & 24089 & 18647 & $\mathbf{2 2 . 5}$ & 21000 \\
\hline 2-B & 20850 & 14276 & $\mathbf{3 1 . 5}$ & 17468 \\
\hline 2-C & 21298 & 15117 & $\mathbf{2 9 . 0 2}$ & 18290 \\
\hline
\end{tabular}

\section{Conclusions}

The reliability of a voltage control devices like load tap-changers is adversely affected when exposed to conditions of rapid voltage fluctuations. Such devices, while effective in mitigating slow variations in voltage, owing to their mechanical nature could be stretched to their limits of operation due to the interaction of the distributed energy resources with the power grid. This article is the initial step toward developing a thorough understanding of the mechanisms of degradation of conventional voltage control devices due to frequent changes in load power injections. The future work in this direction will tie the results obtained in sections 7 and 8 with the aging models presented in section 3 . This information will be used to ascertain the likelihood of failure, under conditions of high operating stress.

The overall objective is to evaluate the cost of operating the voltage control assets incurred by the utilities in conditions of accelerated aging due to variability of distributed energy sources. We plan to develop a cost-benefit analysis of voltage control devices and look for viable solutions that would either alleviate the excessive stresses or supplant the existing mechanical voltage control assets in the distribution systems. To that end, this paper outlines some procedures to conduct such studies and offers a solution in the form of a capacitor-less D-STATCOM.

\section{Acknowledgment}

This publication was made possible by NPRP grant \# 9-204-2-103 from the Qatar National Research Fund (a member of Qatar Foundation). The statements made herein are solely the responsibility of the authors.

\section{References}

[1]International Energy Agency, "Snapshot of Global Photovoltaic Markets - 2018," 2018.

[2]M. M. Begovic, I. Kim, D. Novosel, J. R. Aguero, and A. Rohatgi, "Integration of photovoltaic distributed generation in the power distribution grid," in Proceedings of the Annual Hawaii International Conference on System Sciences, 2012.

[3]F. Katiraei and J. R. Agüero, "Solar PV integration challenges," IEEE Power Energy Mag., 2011.

[4]M. Begovic and A. Peerzada, "Impact of Large Distributed Solar PV Generation on Distribution Voltage Control," in Proceedings of the 52nd Hawaii International Conference on System Sciences., 2019, pp. 3473-3482.

[5]G. K. Ari and Y. Baghzouz, "Impact of high PV penetration on voltage regulation in electrical distribution systems," in 3rd International Conference on Clean Electrical Power: Renewable Energy Resources Impact, ICCEP 2011, 2011.

[6]R. Yan, B. Marais, and T. K. Saha, "Impacts of residential photovoltaic power fluctuation on on-load tap changer operation and a solution using DSTATCOM," Electr. Power Syst. Res., 2014.

[7]X. Zhang, E. Gockenbach, V. Wasserberg, and H. Borsi, "Estimation of the lifetime of the electrical components in distribution networks," IEEE Trans. Power Deliv., 2007.

[8]X. Zhang and E. Gockenbach, "Assessment of the actual condition of the electrical components in medium-voltage networks," IEEE Trans. Reliab., 2006.

[9]J. D. Baldwin and C. A. Paul, "Comparative statistical analysis of fatigue crack growth data," in 51th Society for Machinery Failure Prevention Technology (MFPT Society), 1997, pp. 379-388.

[10]Q. Chen and D. M. Egan, "A bayesian method for transformer life estimation using Perks' hazard function," IEEE Trans. Power Syst., 2006.

[11]A. Bossi, J. E. Dind, J. M. Frisson, U. Khoudiakov, H. F. Light, and et. al., "An International Survey of Failures in Large Power Transformers in Service," Final report of the CIGRE Working Group 12.05, Electra. 1983.

[12]A. Drobyshevski, V. Sokolov, and S. Tsurpal., "Reliability problems with large power transformers and shunt reactors. typical failure modes and failure causes.," in Transformer Reliability and Transients Moscow Russia, pp. 20-23.

[13]P. de Klerk, M. Minhas, and J. Reynders, "Failures in power system transformers and appropriate monitoring techniques.," in High Voltage Engineering Symposium, p. No 467.

[14]A. van Schijndel, "Power Transformer Reliability Monitoring," Eindhoven University of Technology, 2010.

[15]J. J. Grainger and S. Civanlar, "Volt/Var Control on Distribution Systems with Lateral Branches Using Shunt Capacitors and Voltage Regulators Part II: The The Solution Method," IEEE Trans. Power Appar. Syst., 1985.

[16]K. P. Schneider et al., "Analytic Considerations and Design Basis for the IEEE Distribution Test Feeders," IEEE Trans. Power Syst., 2018.

[17]"http://www1.eere.energy.gov/buildings/commercial/ref_building s.html." E. Wilson, [Online]. Available: http://www1.eere. energy. gov/buildings/commercial/ref_buildings.html.

[18]C. A. Gueymard, "From Global Horizontal To Global Tilted Irradiance: How Accurate Are Solar Energy Engineering Predictions in Practice?," in ASE Society Conference (SOLAR 2008), 2008. 